\title{
Painful swelling in the thigh: diabetic muscle infarction
}

\author{
Ami Schattner MD, Taiba Zornitzki MD, Meital Adi MD, Joshua Friedman MD
}

The case: A 52-year-old woman was admitted with painful swelling in her left thigh. She had a 12-year history of type 2 diabetes mellitus and hypertension, and she was a smoker (40 pack-year smoking history). At her last examination, her $\mathrm{HbA}_{1 \mathrm{c}}$ level was $7.7 \%$, her serum total cholesterol level was 8.65 (normal < 5.17) $\mathrm{mmol} / \mathrm{L}$ and her triglyceride level was 3.77 (normal < 2.26) $\mathrm{mmol} / \mathrm{L}$. She had diabetic retinopathy and microalbuminuria, and she had a myocardial infarction 12 years earlier. Two months before admission, the patient underwent a left femoropopliteal bypass for peripheral vascular disease. Her medications at the time of admission included acetylsalicylic acid, enalapril, metformin, glibenclamide and simvastatin.

On admission, the patient complained of continuous severe pain in her anterior thigh that had developed gradually. The pain was not reduced by nonsteroidal antiinflammatory drugs taken orally. She reported that the pain was very intense, and that it was present at rest but became worse with movement. The pain had increased over the 4 weeks before presentation. She denied any trauma, fever or chills. The patient had lost $4 \mathrm{~kg}$, and she was unable to walk because of the pain.

A physical examination showed a firm swelling in the anterior part of her thigh. An area that measured $10 \times 5 \mathrm{~cm}$ was hard and felt warm, and there was mild erythema and tenderness. The patient's muscle strength and motion were markedly limited by pain.

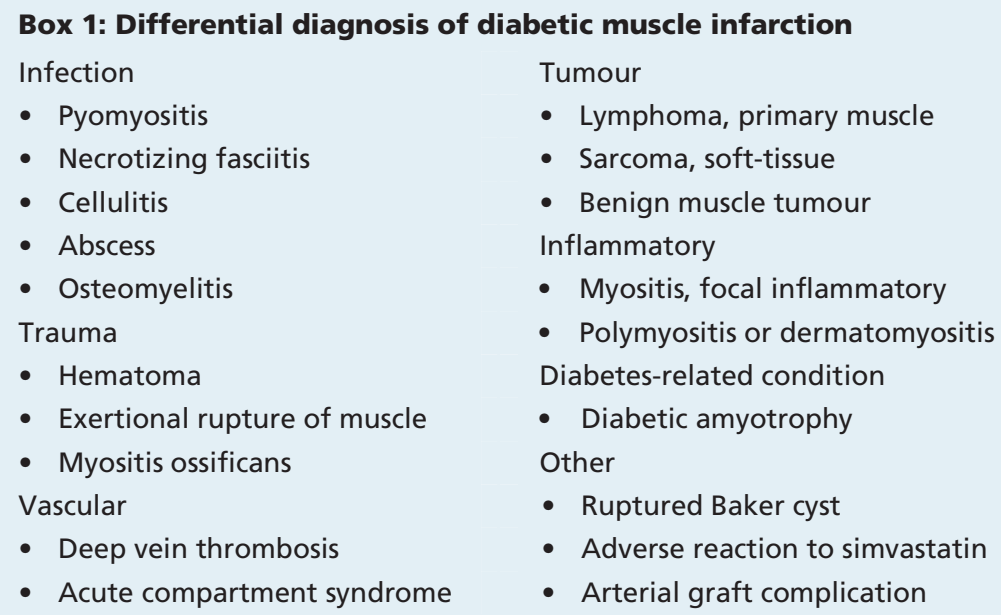

\section{Key points}

- Patients with longstanding diabetes and microvascular complications may develop diabetic muscle infarction.

- Patients usually present with a painful, tender muscle mass, often in the thigh.

- Constitutional symptoms are usually absent.

- Levels of muscle enzymes may not be increased but levels of acute phase reactants often are.

- The diagnosis is clinical, and an MRI can confirm the diagnosis.

- Diagnostic tests (imaging, laboratory investigations) can be used to rule out other causes.

- Slow, spontaneous resolution usually occurs; muscle biopsy or surgery can delay recovery.

Her acute phase reactants were elevated: her erythrocyte sedimentation rate was 153 (normal 0-30) $\mathrm{mm} / \mathrm{h}$, and her C-reactive protein level was 115 (normal $0.2-3.0$ ) $\mathrm{mg} / \mathrm{L}$. She was anemic (hemoglobin $6.2 \mathrm{mmol} / \mathrm{L}$ ) and had mild leukocytosis (11.2 [normal 3.8-9.8] $\times 10^{\%} / \mathrm{L}$, with $78 \%$ polymorphonuclear cells). She also had a mildly reduced level of serum albumin (31 [normal 41-53] g/L) and a normal globulin level (30 [normal 20-35] g/L). The results of liver function tests were normal. Doppler ultrasonography showed no abscess or deep vein thrombosis. Her femoropopliteal bypass graft and larger arteries were patent. Magnetic resonance imaging showed increased signal intensity on $T_{2}$-weighted images of her left quadricep, with diffuse subcutaneous and fat edema. There was extensive swelling of the quadriceps vastus medialis and rectus with intermuscular edema (Figure 1, Figure 2).

Despite 3 weeks of bed rest, opiates, enoxaparin and clopidogrel, there was only a slight clinical improvement. However, her Creactive protein level gradually decreased. An open muscle biopsy was performed to rule out a tumour. Instead, diabetic muscle infarction was confirmed (Figure 3). One week after the biopsy, there was significant worsening of her pain and severe edema of

From the Departments of Medicine (Schattner, Zornitzki, Friedman) and Radiology (Adi), Kaplan Medical Centre, Rehovot; and the Faculty of Medicine (Schattner, Zornitzki, Adi, Friedman), Hebrew University and Hadassah Medical School, Jerusalem, Israel 
the whole thigh. After 2 months of bed rest, tramadol for pain and a combination of clopidogrel and low-molecularweight heparin, all of her complaints and physical findings gradually dissipated. The results of her blood tests normalized. The patient resumed working and was well 10 months later.

Our patient presented with long-term intense leg pain and dysfunction, a large tender mass in her thigh, elevation of acutephase proteins and a prolonged unrelenting course. A long list of differential diagnoses was proposed (Box 1). Although diabetic muscle infarction seemed the most likely diagnosis, a muscle biopsy was judged necessary to rule out a primary muscle tumour. The biopsy was followed by clinical deterioration, similar to that reported in other cases.

About 100 cases of diabetic muscle infarction have been reported since the first report in $1965 .{ }^{1,2}$ Thus, idiopathic mus-
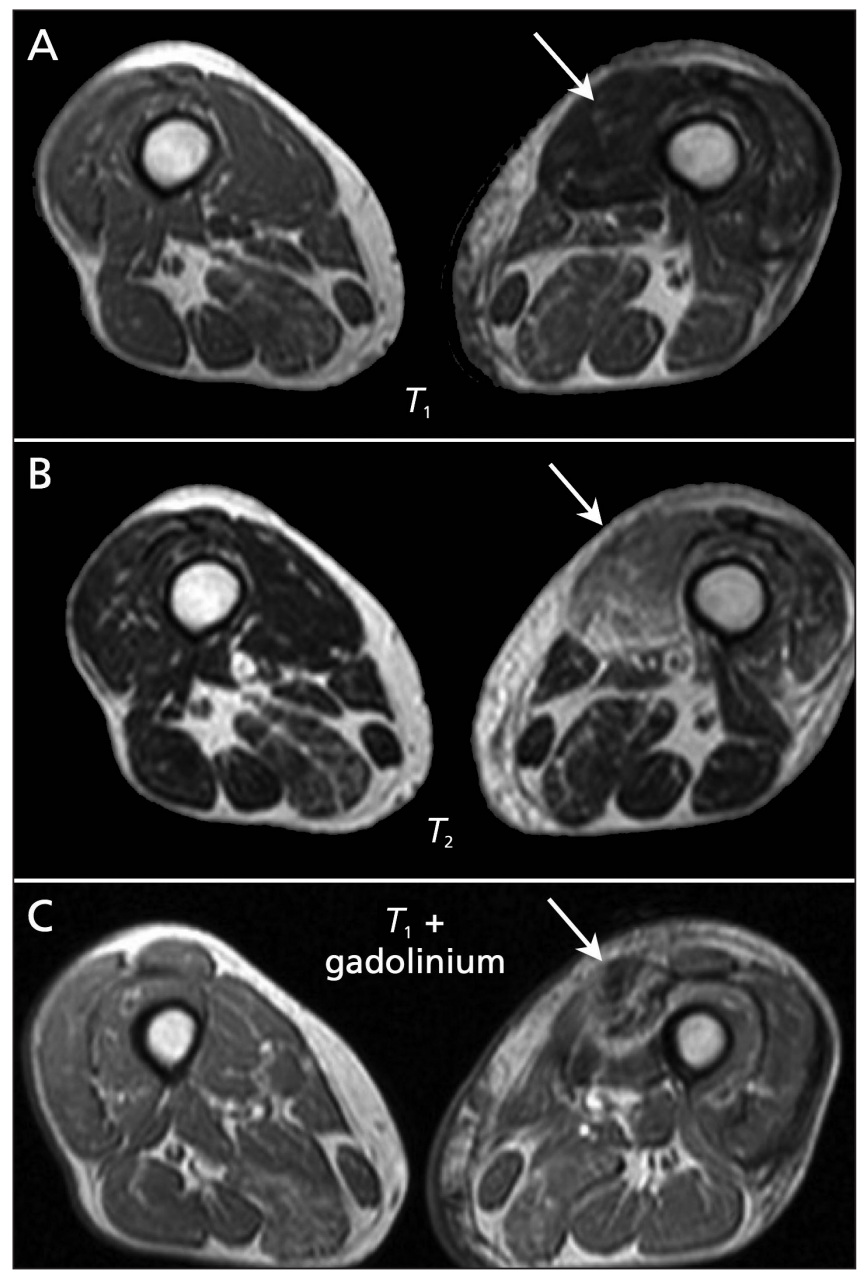

Figure 1: Magnetic resonance imaging of the patient's thighs. A: $T_{1}$-weighted images showing swelling and hypointensity (arrow) of the involved muscle. B: $T_{2}$-weighted images showing diffuse, heterogeneous signal in the involved muscle (arrow), suggesting edema. C: A focal area of heterogeneously enhanced mass (arrow) with peripheral enhancement was visible after adminstration of gadolinium. cle infarction is rare and occurs specifically among patients with diabetes. Although the exact pathogenesis is unknown, it is assumed to be a microvascular complication of diabetes. Most affected patients have a long history of diabetes (either type 1 or type 2 ) and about $97 \%$ have other microvascular complications, most often nephropathy. ${ }^{2}$

Patients typically present with intense, nonremitting and disabling pain in a lower limb with associated edema and mass. Normal temperature and normal levels of muscle enzymes are also typical. ${ }^{2}$ Muscles in the thigh are affected 4 times more frequently than the calf. The quadriceps are the most commonly affected muscles. Involvement of the arms is rare.

Magnetic resonance imaging (MRI) is the diagnostic test of choice, and $T_{2}$-weighted axial plane images are useful for diagnosis. ${ }^{2,3}$ Other diagnostic modalities may help to rule out other diagnoses, rather than help to make the correct diagnosis of diabetic muscle infarction. The clinical presentation and MRI findings are distinctive (Figure 1, Figure 2); thus, a biopsy is rarely indicated. Muscle biopsy and surgery should be avoided despite the severe presentation because these interventions can prolong resolution or temporarily worsen the patient's symptoms.

Whether this rare complication of diabetes can be prevented is unknown. Diabetic muscle infarction is selflimiting, and full recovery can be expected over time. Recommendations for treatment are based on limited evidence and expert opinion. We recommend supportive treatments, such

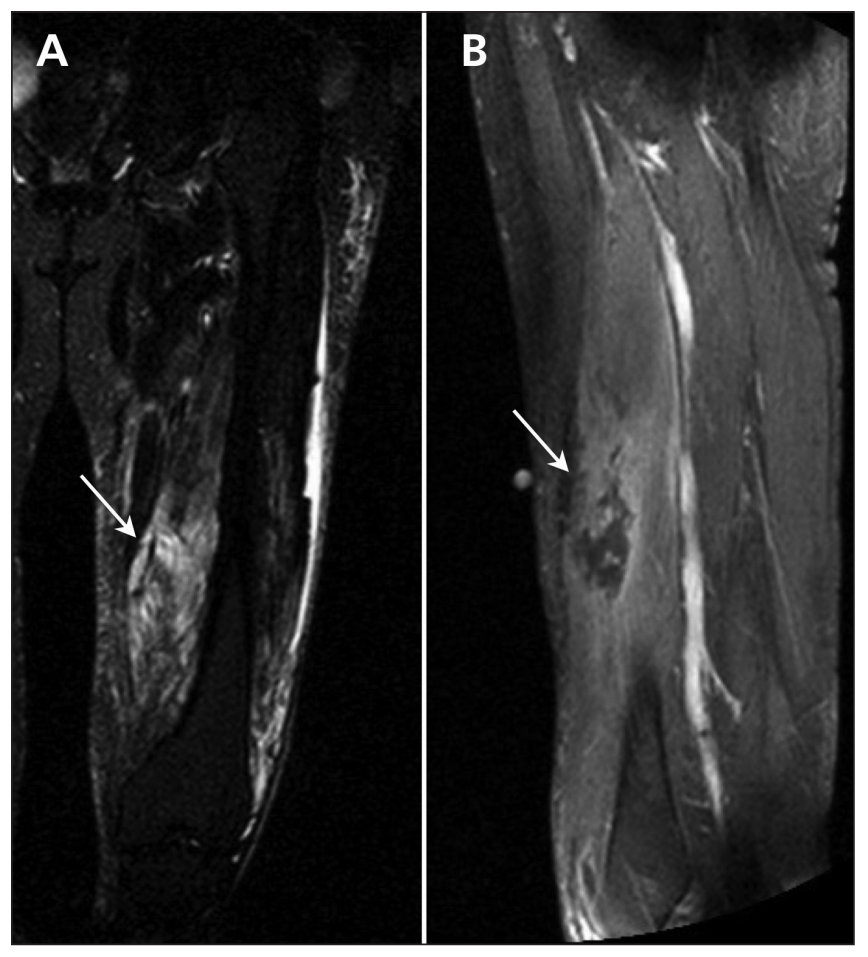

Figure 2: Magnetic resonance imaging of the patient's thigh A: Coronal image showing diffuse, heterogeneous, intense signal of the involved muscle (arrow), which suggests edema. B: Sagittal image showing a heterogeneously enhanced mass (arrow) with peripheral enhancement after adminstration of gadolinium. 


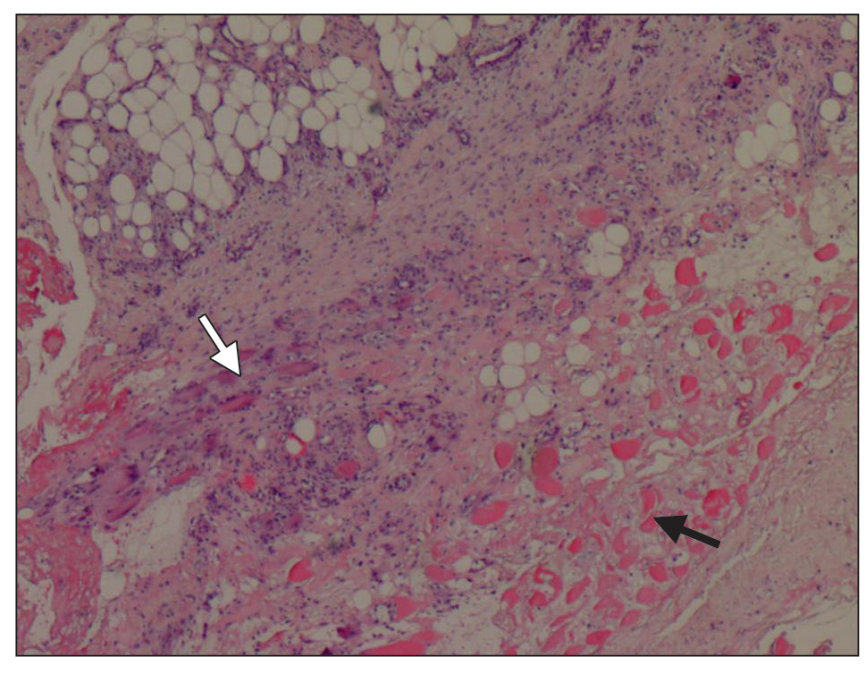

Figure 3: Muscle biopsy showing ischemic skeletal muscle fibres with areas of necrosis (black arrow), reparative changes with myofibre regeneration (white arrow), signs of fibrosis, fat necrosis and chronic inflammation. Atherosclerotic changes are seen in medium-sized vessels and fibrin thrombi are seen in the small vessels (hematoxylin-eosin stain, original magnification $\times 40$ ).

as bed rest, analgesics and, if required, a cautious use of antiinflammatory medications. As a possible microvascular complication of diabetes, antiplatelets are theoretically effective, and advice such as optimizing glycemic control and smoking cessation seems prudent. In one analysis of cases reported from several sources, patients who underwent surgery had a recovery period that was more than double that of those who received conservative treatments such as bed rest, analgesics and anti-inflammatory medications ( 13 weeks v. 5.5 weeks, $p<0.05)^{4}$

Unfortunately, diabetic muscle infarction recurs in about one-half of patients. ${ }^{1,4}$ The overall prognosis for these patients may be related to the severity of their underlying diabetes and the degree of macrovascular and especially microvascular complications.

In conclusion, diabetic muscle infarction is a rare complication of diabetes. The incidence is likely to increase because of the increasing global prevalence of diabetes. Physicians should consider diabetic muscle infarction in the differential diagnosis for patients with diabetes who have a painful, swollen muscle. Conservative treatment strategies should be chosen over aggressive invasive measures when possible.

Competing interests: None declared.

\section{REFERENCES}

1. Kapur S, Brunet JA, McKendry RJ. Diabetic muscle infarction: case report and review. J Rheumatol 2004;31:190-4.

2. Trujillo-Santos AJ. Diabetic muscle infarction. An underdiagnosed complication of long-standing diabetes. Diabetes Care 2003;26:211-5.

3. Kattapuram TM, Suri R, Rosol MS, et al. Idiopathic and diabetic skeletal muscle necrosis: evaluation by magnetic resonance imaging. Skeletal Radiol 2005; 34:203-9.

4. Kapur S, McKendry RJ. Treatment and outcomes of diabetic muscle infarction. $J$ Clin Rheumatol 2005;11:8-12.

Teaching cases are brief case reports that convey clear, practical lessons. Preference is given to common presentations of important rare conditions, and important unusual presentations of common problems. Articles start with a brief summary (100 words) outlining the case and its relevance to a general audience. The case presentation follows (500 words maximum) as well as a discussion of the underlying condition (1000 words maximum). Up to 5 references are permitted and visual elements (e.g., tables of the differential diagnosis, clinical features or diagnostic approach) are encouraged. Written consent from patients for publication of their story is a necessity and should accompany submissions. See information for authors at www.cmaj.ca.

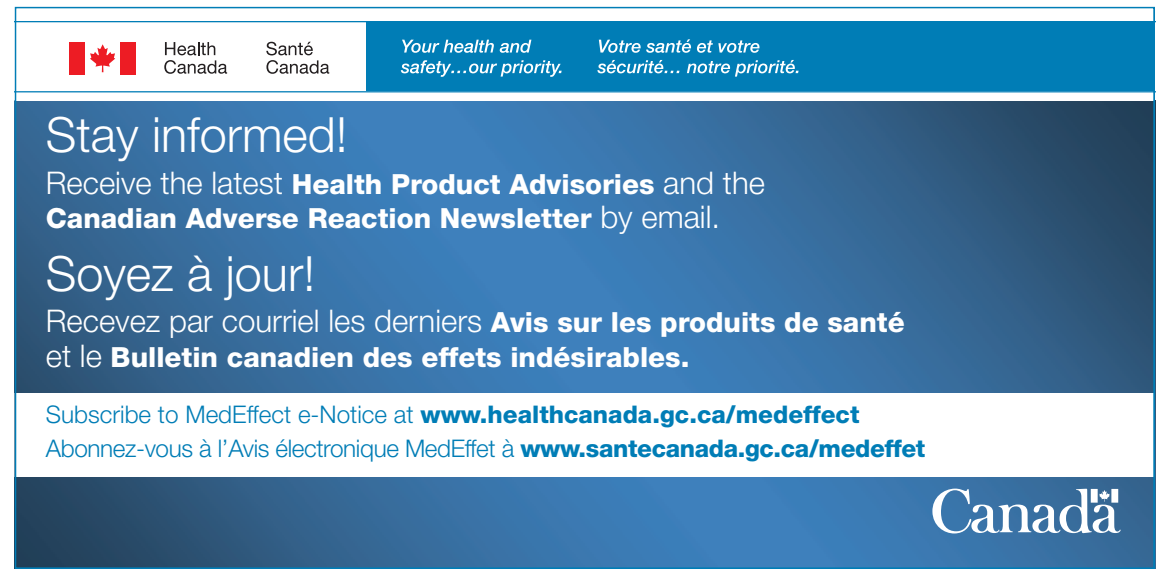

